\title{
HCCA for Wireless Mobile Coverage Networks
}

Chongdeuk Lee*

\author{
Division of Electronic Engineering, Chonbuk National University, Korea; cdlee1008@jbnu.ac.kr
}

\begin{abstract}
Objectives: To reduce performance degradation caused by traffic congestion and improves the network service quality using HCCA (Hybrid Congestion Control Algorithm). Methods/Statistical Analysis: Congestion provides the source of packet loss, throughput degradation and increased power consumption. The performance of media streaming under wireless mobile coverage networks depends on congestion control metric to avoid the traffic load. In this paper, we propose a new HCCA (Hybrid Congestion Control Algorithm) to efficiently control the traffic congestion for wireless mobile coverage networks. To address the traffic congestion problem, the proposed method considers a hybrid congestion control algorithm, based on a RBM (Rate-Based Method) and a BBM (Buffer-Based Method) to adjust the stream packet rate and sufficiently utilize the buffer cache for client nodes. Findings: The role of congestion detection in HCCA is affected by the network performance and QoS (Quality of Service) for each client node. To perform the above process, each client node makes use of its current remaining buffer capacity and traffic capacity. Then, the congestion decision procedure reflects the traffic rate for each client node. As such, each client node use itself congestion rate and neighbors' congestion information to prevent the excessive congestion caused by explosive traffic load. The simulation results show that the proposed method has better performance than the other existing methods. Improvements/Applications: In case of congestion control algorithms, each client nodes does not efficiently control the traffic caused by the explosive data flow and adhesive flow stream. The proposed HCCA have that controls effectively problems generated from network bottleneck.
\end{abstract}

Keywords: BBM, Congestion, HCCA, Mobile Coverage Network, Traffic Load, RBM

\section{Introduction}

With the rapid development and broad application of the internet, wireless communications and personal mobile technology also continue to develop. Mobile devices, such as laptops, smart phones and tablets, allow greater work productivity and convenience. However, higher requirements are demanded for these wireless mobile networks and, because of this, more technical problems may occur. In a wireless mobile network, client nodes forward toward base stations, which are inter-connected with other network devices to access WLANs. For the streaming media service in this environment, managing substantial amounts of streaming media data may be very difficult, and also it is an important issue to address ${ }^{1-3}$. The congestion control problem is one of these important issues. Many studies have focused on the congestion problem as a major bottleneck to network efficiency.
Recently, TCP has been investigated as a mechanism for controlling media data in a wireless network. TCPbased protocols, however, have problems with frequent disconnection ${ }^{1}$ and traffic error ${ }^{2,3}$, because of the sensitivity to delays and network congestion. When a streaming media service is performed in a wireless channel, the quality of streaming may be affected, due to problems with explosive traffic load, constricted resources, and bandwidth constraints. In circumstances of constricted bandwidth and long delay in a wireless channel, TCPbased stream packet service has problems in reliability due to traffic congestion and jitter delay. The HCCA addressed in this paper is a means to overcome these constraints.

Many TCP-friendly control methods have been proposed to solve the congestion problem $\frac{4,5}{5}$, including window-based schemes ${ }^{4}$ and rate-based schemes. Ratebased schemes may be classified as probe-based schemes

${ }^{*}$ Author for correspondence 
or equation-based schemes ${ }^{\underline{6}}$. Equation-based schemes ${ }^{\underline{6}}$ provide high transmission rates under constant conditions of packet size, packet loss rate, and RTT (Round Trip Time) ${ }^{2}$. TFRC (TCP-friendly Rate Control $)^{6}$ is an equation-based scheme that is efficient for unicast flow of packets with constant size. If a wireless channel does not control traffic congestion appropriately, the QoS (Qualityof-Service) performance may degrade, since the packet size of media data is variable. Traffic congestion control in the wireless coverage network plays an important role for throughput, as it enhances streaming QoS and supports network balance and reliable responsiveness $\frac{7.8}{}$. To realize streaming QoS , proposed a TRM-based multimedia streaming optimization method. This method improves the streaming QoS by considering minimum bandwidths, maximum bandwidths, and transmission rates in multimedia application domains.

Traffic congestion control generally follows three step ${ }^{10-12}$ : congestion detection, congestion notification, and rate-adjusting. An important key metric of congestion control is to decide whether it can achieve the following performance objectives: 1 . Possess power consumption-efficiency which is required in order to extend system lifetime; 2. Consider traditional QoS metrics such as the packet-loss ratio, jitter delay and throughput; 3 . Have dynamic adaptability (the protocol must be able to adapt to network changes and environment changes, and be able to adapt to both partial network congestion as well as the entire local network congestion); 5. Be able to predict or quickly detect network congestion, and relieve congestion in a relatively short period of time, to avoid congestion diffusion; and 6. Adjust the congestion rate so that client nodes can support the maximum traffic throughput.

This paper proposes a new HCCA (Hybrid Congestion Control Algorithm) to improve the throughput and network QoS in the wireless coverage network. The proposed HCCA considers the congestion detection process, stream packet rate, and remaining buffer capacity in the buffer cache. To acquire the congestion information, each client node makes use of its current remaining buffer capacity and traffic capacity. The congestion decision procedure reflects the traffic rate for each client node. Then, the congestion rate is updated periodically by monitoring the relationship between client nodes and neighbor nodes. As such, each client node use itself congestion rate and neighbors' congestion information to prevent the excessive congestion caused by explosive traffic load.
The rest of this paper is organized as follows. In Section 2, we describe details of the proposed HCCP algorithm while a performance evaluation using the simulation experiments is described in Section 3. Finally we provide our concluding remarks in Section 4.

\section{HCCA (Hybrid Congestion Control Algorithm)}

To efficiently control congestion, this section considers the buffer capacity and the stream packet rate. We first define the buffer capacity of each node by $B$, the remaining buffer capacity by RB and net flow capacity by NS. When the traffic congestion occurs at any node, the congestion rate is represented by $\mathrm{CR}$ and the potential traffic congestion is represented by $\mathrm{TC}_{\mathrm{i}}$. Terminologies for the HCCA algorithm are defined in Table 1.

Table 1. Terminologies for HCCA

\begin{tabular}{|l|l|}
\hline Terminologies & Meaning \\
\hline B & Buffer length of each node \\
\hline RB & Remaining buffer length \\
\hline NS & A net flow capacity \\
\hline CR & Congestion rate \\
\hline TC $_{i}$ & Potential traffic capacity \\
\hline
\end{tabular}

The HCCA (Hybrid Congestion Control Algorithm) is comprised of two procedures: the congestion detection procedure and the stream packet rate adjustment procedure. The protocol is used only on the local region among several nodes and each node makes use of its current remaining buffer capacity and net flow capacity to calculate its congestion rate. Additionally, the congestion rate is updated between neighbors at each unit time; each client node may use this congestion rate to prevent congestion.

\subsection{Congestion Detection Procedure}

Since each client node may transmit one or more stream packets toward neighbor nodes and the base station, every client node can meet a heavy congestion load. Congestion in this direction mainly may occur when the stream packets forward explosively toward neighbor nodes and the base station. When a node meets the congestion, it will be consumed a wireless resources and time $\frac{13,14}{14}$. In this case, the congestion rate reference a time slot $\mathrm{T}$. The time slot $\mathrm{T}$ should not be too long, not too short. If $\mathrm{T}$ is too short, 
it will meet high context-switching problem caused by the frequent congestion detection. In contrast, if $\mathrm{T}$ is too long, the congestion will meet before time is finished. It results in degrade the performance of congestion control.

To detect the congestion efficiently, client nodes has two types of node search steps: First searching step is toward the base station, second step is toward adjacent nodes. If the stream packet rate incoming from the upstream neighbors is less than the occupying stream packet rate in the buffer cache, it can forward the stream packets without the congestion. The buffer cache for stream packets has an available caching capacity and congestion will not meet on it in the next step.

In Figure 1, node $i$ s upstream neighbor is node $x$ and its downstream neighbor is node $y$; the data rate $r_{i, y}=15$ and $r_{x, i}=12$. So congestion will not meet at node $i$ in the next step, and then congestion control does not meet any more.

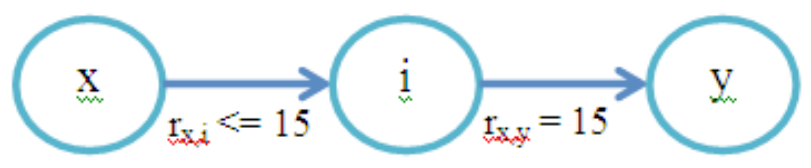

Figure 1. Example of downstream data rate is larger than or equal to stream data rate.

In Figure $2(\mathrm{a})$, the stream packet rates are: $r_{x, i}=15$, $r_{i, y}=10$; so the protocol expects a likelihood of congestion at node $i$. There are two situations: First, node $i$ does not fully utilize the downstream bandwidth; the protocol can promote the downstream packet rate until it is equal to the upstream packet rate. Second, node $i$ fully utilizes the downstream bandwidth. It can not boost the downstream packet rate. Thus, node $i$ will search the neighbor nodes to see if the buffer capacity has been used. In Figure 2(b), if the remaining buffer capacity of neighbor node $z$ equals the buffer capacity when it is in a no-load state, then this implies that the stream packet received from the upstream neighbors are all sent to the downstream neighbors. Because the buffer capacity of node $z$ has not been used, node $i$ will send the remaining stream packet to node $z$. If the buffer capacity of node $z$ has already been used, node $i$ will not send stream packet to node $z$, but holds the stream packet in its own buffer cache. At the same time, node $i$ suppresses the upstream neighbors to slow down their stream packet rate. We say that the buffer cache of node $i$ is in a light-load state.

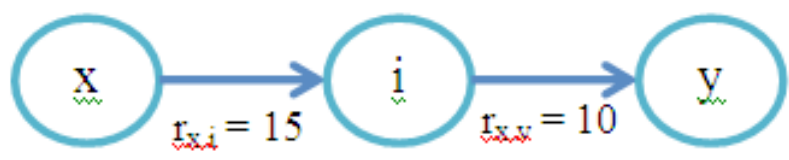

(a)

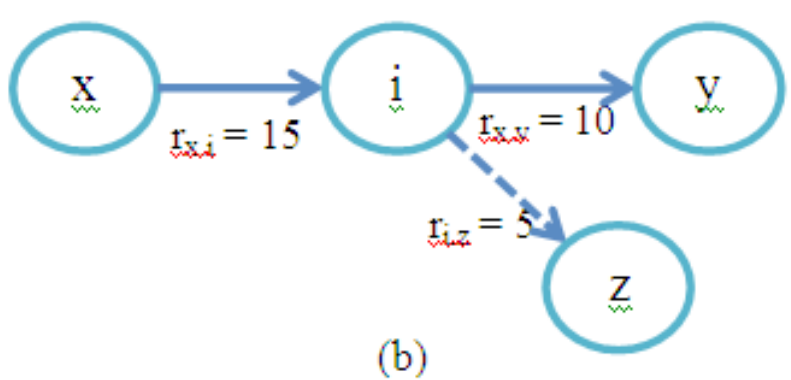

Figure 2. Example of upstream packet rate is larger than or equal to downstream packet rate.

When the spare cache capacity of node $i$ in the next step is less than the size of the stream packets incoming from the forwarding nodes, the buffer cache for node $i$ meets a heavy-load state. If a heavy load is not controlled appropriately, congestion still may meet in the next step. To prevent this potential congestion, the congestion control process has to consider the proper stream packet rate for its forwarding nodes, according to their congestion rates.

In order to prevent the explosive traffic load, a node $i$ have to measure the maximum amount of traffic rate from its forwarding nodes at any time. Let $\mathrm{R}_{\mathrm{x}, \mathrm{i}}$ be the maximum stream packet from node $\mathrm{x}$ to $\mathrm{i}$ at any time $\mathrm{T}$. Then a receive stream packet $R S_{i}$ is the packet processing value of node $i\left(r_{i}\right)$ plus stream packets of forwarding nodes for node $i$ at any time $\mathrm{T}$ is defined as the following.

$\boldsymbol{B}_{i}=\left(r_{i}+\sum_{x \in U_{i}} R_{x, i}\right) \times T, \forall x, i \in N$

We do not consider the downstream packet rate from node $i$ because, when congestion meets, we are not sure whether node $i$ can send stream packet to its downstream neighbors.

In order to show the traffic load rate for congestion, a congestion rate $\mathrm{CR}_{\mathrm{i}}$, is considered, $\mathrm{CR}_{\mathrm{i}}$ is the extra cache capacity minus the stream packet rate of each node $i$ at any time $\mathrm{T}$ is defined as the following. 


$$
C R_{i}=R B_{i}-R S_{i}, \forall i \in N
$$

If the value $\mathrm{CR}_{\mathrm{i}}$ is smaller than 0 , node $i$ have the heavy-load state. Therefore, to reduce the heavy-load state, $\mathrm{CR}_{\mathrm{i}}$ must have a proper stream packet capacity. If $\mathrm{CR}_{\mathrm{i}}$ violate the conditions, it results in meet the congestion and degrade the system perform.

\subsection{Stream Packet Adjustment Procedure}

The nodes have to detect the buffer cache occupation and stream packet capacity as fast as possible when any node i meet the congestion in forwarding the media stream packets toward adjacent nodes or the base station. If $\mathrm{CR}_{\mathrm{i}}$ are larger than or equal to 0 , the stream packet of $i$ will have the week-load state. Then, it will process the next procedure. However, if $\mathrm{CR}_{\mathrm{i}}$ is smaller than 0 , node i will have the heavy-load state. Therefore, it need to consider the stream packet capacity and occupying buffer cache to avoid the congestion problem; let this stream packet rate be equal to or greater than the absolute value of $\mathrm{CR}_{\mathrm{i}}$. Node $i$ is one of the upstream nodes of node a. Therefore, the more negative the value of $\mathrm{CR}_{i}$, the larger the stream packet capacity should be adjusted node i. If $\mathrm{CR}_{\mathrm{i}}(\mathrm{a})<0$, $\mathrm{SUM}_{\mathrm{a}}$ can be defined as:

$$
S U M_{a}=\sum_{i \in U_{a}}\left|C R_{i}(a)\right|, \forall C R_{i}(a)<0
$$

We suppose that the bandwidth is fixed, and $\mathrm{TC}_{\mathrm{i}}$ is the minimum amount of flow size. If node $i$ have enough buffer capacity to hold the stream packet from its upstream nodes by the full flow rate during a time period $\mathrm{T}, \mathrm{TC}_{\mathrm{i}}$ is the full flow rate from all upstream nodes. But, if there is not enough buffer capacity, $\mathrm{TC}_{\mathrm{i}}$, can be defined as:

$$
T C_{i}=\operatorname{MIN}\left(R B_{i}, \sum_{x \in U_{i}} R_{x, i} \times T\right)
$$

Here, the value $T C_{i}$ represents the metric that node $\mathrm{i}$ occupy stream packets from their adjacent nodes at any time. Node i measure whether its $T C_{i}$ value is satisfied, with $C R_{x}(i)<0 . S T C_{i}=T C_{i}-S U M$ means that $T C_{i}$ mitigate the congestion caused by traffic load from node i. If $T C_{i}>0$, node i will re-allocate stream packet capacity by considering the occupying buffer cache. If $C R_{x}(i)<0$, the traffic load of node i will meet any more. Let $\mathrm{NU}_{\mathrm{i}}$ be the number of upstream neighbors of node i. If any node $i$ meet the new stream packet rate $\mathrm{NR}_{\mathrm{x}, \mathrm{i}}$ from their adjacent nodes, $\mathrm{NR}_{\mathrm{x}, \mathrm{i}}$ is defined as the following.

$$
N R_{x, i}= \begin{cases}\left|C R_{x}(i)\right|+\frac{S T C_{i}}{N U_{i}}, & C R_{x}(i)<0 \\ \left(\frac{S T C_{i}}{N U_{i}}\right), & C R_{x}(i)>0\end{cases}
$$

If $S T C_{i}<0$, the traffic load of node i will not meet the adjustment for stream packet rate from their adjacent nodes. Adjusting only the stream packet rate of node $\mathrm{i}$ cannot stop congestion from happening in the next time period. Therefore, node i need other neighbor nodes to acquire the congestion information for the remaining stream packet. For node $\mathrm{x}, C R_{x}(i)>0$, node i will interrupt the connection between $\mathrm{x}$ to $\mathrm{i}$ in next time period. For node y, since $C R_{y}(i)<0$, it cannot search the other neighbor nodes to help divide the data. Node i will prioritize the division to ensure the proper data rate supply. For node $\mathrm{z}$, $C R_{z}(i)<0$, and it does have other nodes to help divide the data. The remaining potential traffic load of node $i$ is allocated to $\mathrm{z}$, and the remaining stream packet of node $\mathrm{z}$ will be sent to its neighbors. The following example illustrates this situation.

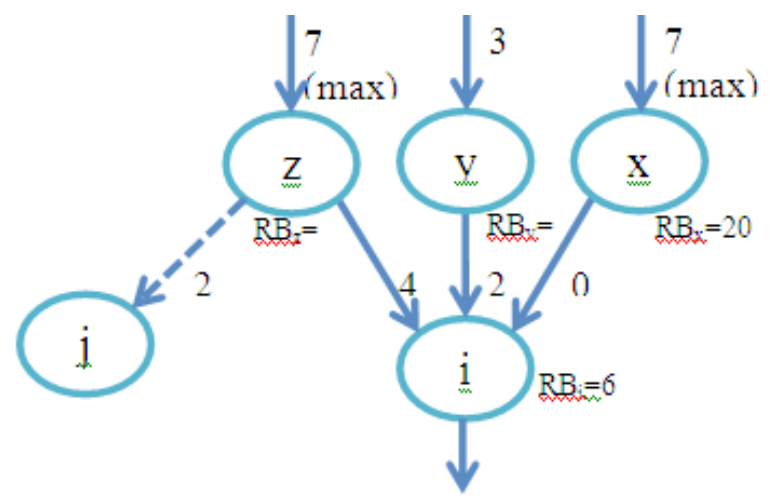

Figure 3. Example of stream packet rate adjustment for $\mathrm{STC}_{i}$ $<0$.

In Figure 3, assume that the maximum amount of flow sizes of node $x, y, z$ and $i$ from upstream are all 7. The extra buffer capacity of nodes $x, y, z$, and i are 20,5, 1 and 6, respectively. Based on (4), the potential traffic load $\mathrm{TC}_{\mathrm{i}}=6$ and the congestion degrees of $\mathrm{x}, \mathrm{y}, \mathrm{z}$ are 13, -2 , -6 , respectively. So $\mathrm{STC}_{\mathrm{i}}=-2$ and node i doesn't satisfy the requirements from their adjacent nodes at any time. The congestion problem will not meet for node $\mathrm{x}$ but for nodes $y$ and $z$; however, node $z$ can find neighbor node $j$ to divert some stream packet. So node i will first consider allocating stream packet rate from node y to node i. The 
extra traffic load of node i will then be allocated to node $z$. The new stream packet rates of $\mathrm{NR}_{\mathrm{x}, \mathrm{i}} \mathrm{NR}_{\mathrm{y}, \mathrm{i}}$ and $\mathrm{NR}_{\mathrm{z}, \mathrm{i}}$ are $0,2,4$, respectively.

\subsection{HCCA Procedure}

The HCCA procedure may be summarized as follows:

For each node $i$ in each time slot $\mathrm{T}$, each node forwards its congestion degree to its adjacent nodes.

Step 1: Congestion Detection Procedure:

- Through downstream and upstream data flow rates of node $i$, determine node $i$ 's state in this time.

- If the upstream data flow rate is larger than the downstream data flow rate, search the neighbor nodes to see whether or not data traffic can diverted.

- From the receive flow size, node $i$ calculates its $C R_{i}$

- Node $i$ measure whether or not congestion will meet at any time $\mathrm{T}$.

Step 2: Stream Packet Rate Adjustment Procedure:

- Node i calculates its values of SUMi, STCi. Note SUMi is the summation of the absolute value of congestion degree of node $i$, when the congestion degree less than 0 . STCi is satisfied with the remaining potential traffic capacity of node $i$.

- If STCi $>0$, node $i$ will assign a new stream packet rate to its adjacent nodes according to (5).

- If $\mathrm{STCi}<0$, each upstream node of node i will search its neighbors nodes to see whether or not stream traffic can be diverted. Then, node i allocates a new stream packet rate to their adjacent nodes according to (5) with the condition that node i must guaranteed the minimum stream packet rate from its upstream nodes.

After a node assign the stream packet rate from their adjacent nodes, it may meet the buffer overflow due to the explosive traffic flow from their adjacent nodes and congestion may occur at this direction. However, these nodes may further suppress their upstream neighbors in a similar way. To mitigate this problem, we have applied the congestion detection procedure and stream packet rate adjustment procedure for the explosive stream packets from the adjacent nodes.

\section{Simulation Results}

To measure the performance of the proposed HCCA algorithm, we consider the following simulation parameters: the bit rate is $1.28 \mathrm{Mbps}$; the flow data size for the congestion is 2.45 Mbyte; and the maximum cell clients for simulation are 35 clients. The other parameters are as follows: the maximum bit rate is $1.57 \mathrm{Mbps}$; the packet size for the flow is $512 \mathrm{kbps}$; and the average link bandwidth is $1.2 \mathrm{Mbps}$. The simulation continues for $560 \mathrm{~s}$ with $0<<1$, and the timeout interval of $(T-\bar{T})$ and $(\bar{T}-T)$ is set to 0.2 s. Here $\bar{T}$ is the duration from mobile client $m$ to base station $n$, and $\lambda$ is the delay rate. The mobile client is assumed to be connected to the IP backbone network using a wireless IP. The performance of the proposed HCCA algorithm was evaluated using the simulation parameters shown in Table 2.

Table 2. Simulation parameters

\begin{tabular}{|l|l|}
\hline Parameters & Value \\
\hline The number of flow data & 5,500 \\
\hline Maximum cell clients & 35 \\
\hline Maximum flow size & 10 Mbytes \\
\hline Simulation time & $560 \mathrm{~s}$ \\
\hline Timeout interval & $0.2 \mathrm{~s}$ \\
\hline Buffer capacity & $0<\mathrm{BC}<100 \%$ \\
\hline Average link bandwidth & $1.2 \mathrm{Mbps}$ \\
\hline Maximum bit rate & $1.5 \mathrm{Mbps}$ \\
\hline
\end{tabular}

The network bandwidth was assumed to operate on a $10 / 100 \mathrm{MHz}$ band with $2 \mathrm{GHz}$ carrier. Each cell was assigned to 35 mobile clients and the location of mobile clients was uniformly random, generated over the area of network. We simulated the proposed HCCA with cell network size $(0,0) \times(250,250)$. To see the performance of the proposed HCCA method, we compared and analyzed the following setups: average congestion rate, traffic overhead, and queuing delay with flow size and buffer capacity. In the simulation process, when an upstream of packets traverses the network, each packet may experience different setups. We define average congestion rate, traffic overhead, and queuing delay as the variance for performance metrics suffered by the upstream flow. The average congestion degree $\left(\mathrm{AC}_{\text {degree }}\right)$, traffic overhead $\left(\mathrm{T}_{\text {overhead }}\right)$, and queuing delay $\left(\mathrm{Q}_{\mathrm{d}}\right)$ are defined as: 


$$
\begin{aligned}
& A C_{\text {degree }}=\frac{T_{\text {buffer capacity }}-\sum_{j=1} \text { upstream } \text { flow data }_{j}}{\sum_{i=1} \text { Queuing }_{\text {flowdata }} \times(\text { (ransmission rate by monitoring })^{\prime}} \\
& T_{\text {overhead }}=\frac{\text { Amount of control traffic }(\text { in } K B)}{\text { Flowdata for upstream } \times B_{r} \times L B_{w}}
\end{aligned}
$$

Where $B w$ is the average bit rate and $B w$ is the average link bandwidth.

$$
Q_{d}=\log _{X}\left(\frac{d_{e}}{d_{u}}\right) \times \lambda
$$

Where $d_{s}$ is the end-to-end delay and $d_{u}$ is the average delay experienced during the upstream flow. For the simulation, all links were assigned separate buffers for each direction, which were capable of queuing up to 50, 100 and 150 packets over WLANs. We analyzed how the proposed protocol performance was affected by the selection of several variable parameters such as the average bit rate, the average link bandwidth, flow size, buffer capacity, and $\lambda$.

Table 3. Simulation results

\begin{tabular}{|l|l|l|l|l|}
\hline Performance & RBM & BBM & TRM & HCCA \\
\hline Throughput & 92.3 & 91.4 & 96.7 & 97.5 \\
\hline Pack Loss Rate & 6.22 & 6.72 & 2.14 & 1.62 \\
\hline Retransmission Rate & 7.44 & 7.93 & 1.52 & 1.27 \\
\hline Jitter Delay Rate & 10.94 & 11.2 & 1.95 & 1.32 \\
\hline
\end{tabular}

Table 3 shows the simulation result for each test case. The results are measured by applying the simulation parameters and average throughput, packet loss rate, retransmission rate, and jitter delay rate over the mobile coverage network. We can see that the proposed HCCA improves network performance when compared to other protocols.

Then we analyzed the congestion degree, traffic overhead, and queuing delay rate to see network performance. The size of the flow data was set between 2 Mbytes and 18 Mbytes, and the average was measured through 10 trials. Figure 4 and Figure 5 show the congestion degree and traffic overhead with flow size, respectively.

Compared with the RBM and BBM methods, we note that the proposed HCCA method doesn't effects the congestion degree significantly. Figure 4 shows the congestion degree value of the base station from the upstream data packets. The proposed HCCA performed better than the other protocols. As shown in Figure 4, BBM and TRM generated similar results. This led us to conclude that, for the buffer-based scheme and transmission rate monitoring scheme, the type of flow have a little effect on congestion variations. However, in the case of the proposed HCCA method, not only does the protocol play a role in congestion variation, but the type of flow is very crucial in minimizing congestion variations. As the size of flow increases, the performance protocol must be capable of controlling a large amount of data flow efficiently, without raising much congestion variation.

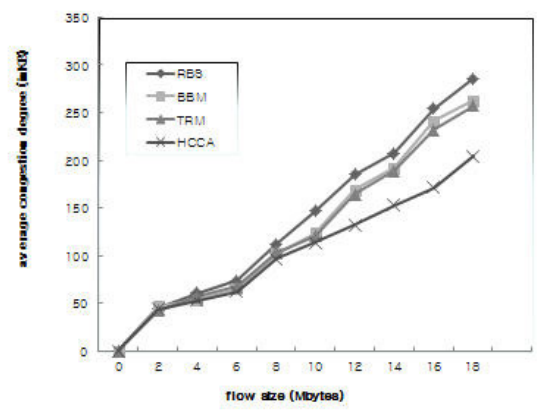

Figure 4. Average congestion degree with flow size.

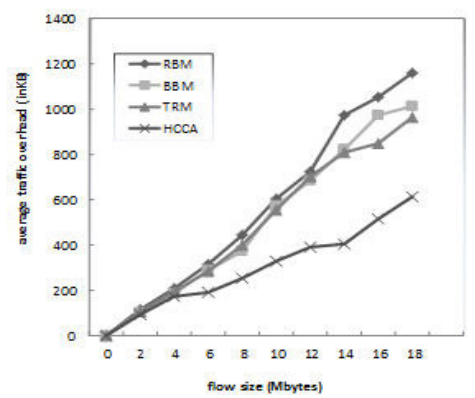

Figure 5. Average traffic overhead with flow size.

Figure 5 shows an average traffic overhead with flow size. As shown in Figure 5, the proposed HCCA method generated lower traffic overhead than the other protocols. The main reason for this is the combination of buffer capacity and rate that the HCCA uses; further, the HCCA method monitors traffic information and flow data packets periodically. In our simulations, this timeout interval was set to 0.2 seconds. The value of this time out interval is very important. A lower timeout interval setup will request the upstream frequently but also the performance of system will degrade rapidly. On the other hand, the larger value of time out interval will not only result in retransmission for upstream but will also result in increase of delay time. Therefore, the proposed HCCA generated a lower traffic overhead than the other protocols. The tuning of this parameter online is an area of future research. 
Figure 6 shows the corresponding rates of the queuing delay for each protocol, and Figure 7 shows average congestion rate with buffer capacity. The queuing delay rate for the HCCA method was relatively lower than the other protocols. As shown in Figure ), the TRM protocol applied a rate similar to the one created by the RBM technique; but the queuing delay rate was much higher. When the delay rate is 0.9 , the average queuing delay rate for the proposed HCCA method was about 30\% lower than the packet processing delay rate experienced using the BBM method. The reason for this is the decrease in the time spent by each packet in the queue. However, if the time spent in processing a data flow packet is much more, this degrades system performance. Also, if the queuing delay in processing a data flow packet becomes too large, the network will experienced additional traffic overhead. Thus, the delay rate plays an important in processing the performance for HCCA and the excessive delay rate can affect the performance.

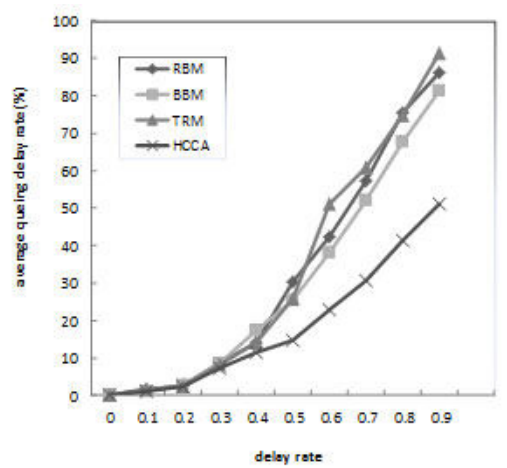

Figure 6. Average queuing delay rate with delay rate.

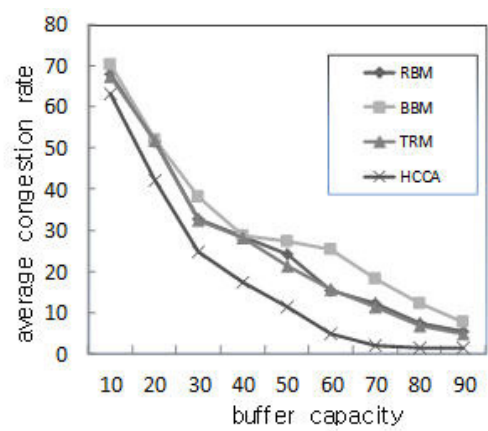

Figure 7. Average congestion rate with buffer capacity.

\section{Conclusions}

In this paper, we have proposed a new Hybrid Congestion Control Algorithm (HCCA) to efficiently control the traf- fic congestion in wireless mobile coverage networks. The proposed HCCA controls mixed media over upstream and also improves the streaming QoS. The main contributions of this paper are summarized as follows: 1 . The HCCA algorithm monitors the network to efficiently adjust the data rate between the available buffer cache and link utilization. 2 The HCCA algorithm considers the characteristics of different types of media and different request patterns, which can improve system performance such as the throughput, packet loss rate, retransmission rate, and jitter delay rate for multiple types of media. 3 . The HCCA approach reduces traffic overhead by adapting to network conditions and media characteristics. Moreover, a transmission rate monitoring strategy and buffer caching strategy fully utilizes both continuous and discontinuous media characteristics. 4. Finally, the hybrid algorithm efficiently controls the upstream between node and base station among different type of requests using a performance metric that efficiently measures the traffic overhead between cell clients and coverage networks, based on media characteristics Simulation results showed that the proposed HCCA strategy can achieve high performance for media upstream and also improve the overall network quality. Simulation results using mixed media with multiple priorities and different request patterns demonstrated that the proposed HCCA method adapts to traffic variations and achieves good quality under wireless coverage network conditions.

\section{Acknowledgment}

This research was supported by Basic Science Research Program through the National Research Foundation of Korea (NRF) funded by the Ministry of Education (No.2013R1A1A4A01005033)

\section{References}

1. Park MY, Chung SH. A simulation-based study on spurious timeouts and fast retransmits of TCP in wireless networks. 3rd International Joint Conference on Computational Science and Optimization (CSO); 2010. p. 273-7.

2. Zhu P, Zeng W, Li C. Joint design of source rate control and QoS-aware congestion control for video streaming over the internet. IEEE. 2007; 9(2):366-76.

3. Sastry NR, Lam SS. CYRF: A theory of window-based unicast congestion control. IEEE. 2005; 13(2):330-42.

4. Rejaie R, Handley M, Estrin D. RAP: An end-to-end ratebased congestion control mechanism for real-time streams 
in the internet. Proceedings of the 18th Annual Joint Conference of the IEEE Computer and Communications Society (INFOCOM'99); 1999. p. 1337-45.

5. Handley M, Floyd S, Padhye J, Widmer J. TCP-Friendly rate control (TFRC): Protocol specification. IETF RFC. 2003: 3448.

6. Fu Z, Zerfos P, Luo H, Lu S, Zhang L, Gerla M. The impact of multihop wireless channel on TCP throughput and loss. Proceedings of IEEE Information Computing; 2003. p. 1744-53.

7. Wang B, Kurose JF, Shenoy PJ, Towsley DF. Multimedia streaming via TCP: An analytic performance study. ACM Trans Multimedia Computing. 2008; 4(2):908-15.

8. Wan CY, Eisenman SB, Campell AT. CODA: Congestion detection and avoidance in sensor networks. Proceeding of ACM Sensys; California, USA. 2003. p. 266-79.

9. Lee CD, Jeong TW, Ahn JY. TRM-based multimedia streaming optimization scheme in wireless networks. International Journal of Innovation Computing and Information Control. 2011; 7(4):1199-209.
10. Park MY, Chung SH. Detecting TCP retransmission timeouts nonrelated to congestion in multi-hop wireless networks. IEICE Transaction on Information and System. 2010; 12(1):3331-43.

11. Brahma S, Chatterjee M, Kwit K, Varshney PK. Traffic management in wireless sensor networks: Decoupling congestion control and fairness. Comp Comm. 2012; 35(6):670-81.

12. Lee S, Chung K. Channel quality-based rate adaptation scheme for wireless networks. Information Network. 2008; 1-5.

13. Wang C, Li B, Sohraby K, Daneshmand M, Hu Y. Upstream congestion control in wireless sensor networks through cross-layer optimization. IEEE Journal on Selective Areas in Communication. 2007; 25(4):786-95.

14. Antoniou P, Pitsillides A, Blackwell T, Engelbrecht A, Michael L. Congestion control in wireless sensor networks based on bird flocking behavior. Computing Network. 2013; 57(1):1167-91. 